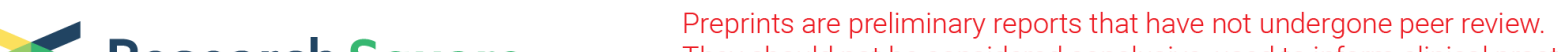 $\begin{array}{ll}\text { Research Square } & \text { They should not be considered conclusive, used to inform clinical practice, } \\ \text { or referenced by the media as validated information. }\end{array}$
}

\section{A Proteomic and Transcriptomic Analysis Reveals That hsa_circ_0004182 Promotes the Expression of Glycerol-3-phosphate Dehydrogenase 1 Like via Targeting mir-155 in Coronary Artery Disease}

\section{Qiaowei Jia}

Jiangsu Province Hospital and Nanjing Medical University First Affiliated Hospital

Wenfeng Ji Jiangsu Province Hospital and Nanjing Medical University First Affiliated Hospital Chenhui Zhao Jiangsu Province Hospital and Nanjing Medical University First Affiliated Hospital Yaqing Zhou Jiangsu Province Hospital and Nanjing Medical University First Affiliated Hospital

\section{Can Hou}

Jiangsu Province Hospital and Nanjing Medical University First Affiliated Hospital Enzhi Jia ( $\square$ enzhijia@njmu.edu.cn )

Department of Cardiovascular Medicine, The First Affiliated Hospital of Nanjing Medical University, Nanjing 210029, Jiangsu Province, China. https://orcid.org/0000-0003-1354-9855

Research article

Keywords: coronary artery disease, ceRNA, proteomic and transcriptomic analysis

Posted Date: September 18th, 2020

DOI: https://doi.org/10.21203/rs.3.rs-72837/v1

License: (c) (i) This work is licensed under a Creative Commons Attribution 4.0 International License. Read Full License 


\section{Abstract}

Background: Coronary artery disease (CAD) is one of the leading causes of mortality in the world, and its early diagnosis remains a huge challenge in clinical medicine. Genetic and environmental factors both contribute to the occurrence and development of CAD. It is reported that the inhibition of mitophagy plays an important role in CAD, while the molecular mechanism is still uncertain. In our previous studies, circulating mir-155 in plasma showed a significant differential expression in CAD populations and controls.

Method: In this study, we detected the expression of RNAs and proteins in human coronary artery samples with genome sequencing. Bioinformatics analysis was conducted to find the binding sites of circRNAs, mir-155 and protein coding genes.

Result: 2135 protein groups, 21703 unique peptides and 1640 circRNAs were identified in the samples. 174 differential expressed proteins and 66 differential expressed circRNAs were screened according to the pathological grading and staging of the samples. GO and KEGG analysis showed that the SDEPs were significantly associated with metabolism, oxidative respiration, and mitochondrial function. Based on the gene-gene interaction and gene-protein interaction analysis, a novel ceRNA network may play a regulatory role in CAD.

Conclusion: Hsa_circ_0004182 may inhibit the expression of mir-155 by acting as miRNA sponges, and repress the post-transcriptional repression of GPD1L mediated by mir-155, and promote GPD1L expression in coronary artery disease.

\section{Introduction}

Coronary artery disease (CAD) is one of the leading causes of mortality in the world. According to the statistics from the World Health Organization (WHO), 40 million people died of non-communicable diseases (NCD) worldwide in 2015 , and $45 \%$ of the death toll resulted from cardiovascular diseases, overnumbered cancer $(22 \%)$, chronic respiratory diseases $(10 \%)$ and diabetes $(4 \%))^{(1)}$. The pathogenesis of CAD is complicated. Early in the disease, lipoprotein particles carrying cholesterol penetrate the blood vessel wall through the injured endothelial cells. Macrophages eliminate cholesterol by phagocytosis and transform into foam cells, which activates the inflammatory process and stimulates the proliferation of smooth muscle cells under the artery wall. The chronic vascular remodeling results in the hemadostenosis and blood stream obstruction, leading to the necrosis and disintegration of deep vascular tissues due to malnutrition. Finally, the formation of atheromatous plaque consisting of disintegrating substances and lipids indicates the occurence of atherosclerosis and $\mathrm{CAD}^{(2)}$.

As early as 1950s, significant familial aggregation was found in CAD in a clinical research ${ }^{(3)}$. With the numerous studies on the genetic susceptibility of CAD, it is accepted now that genetic and environmental factors both contribute to the occurrence and development of $\operatorname{CAD}^{(4)}$. 
MiRNAs are a type of endogenous small non-coding RNAs with a length of approximately 22 nucleotides, which participate in the post-transcriptional regulation of target genes by combining with the $3^{\prime}$

Untranslated Region (3'UTR) of the target mRNAs ${ }^{(5)}$. In our previous study, circulating mir-155 in plasma showed a significant differential expression in CAD populations and controls, which means mir- 155 may play a role in the occurence and development of $C A D^{(6)}$. Mir-155 is one of the extensively studied miRNAs and has been reported to be related to CAD. Studies showed that mir-155 regulates the formation of foam cells by its anti-inflammatory and proinflammatory effects ${ }^{(7)}$. However, the regulatory network of mir-155 in CAD remains unclear. In this study, we detected the expression of RNAs and proteins in human coronary artery samples with genome sequencing and revealed a possible competing endogenous RNA (ceRNA) network in CAD.

\section{Methods}

\section{Samples preparation}

To make the results more convincing, we obtained heart specimen from 3 cases of deceased donars (case 1 is a 64-year-old man with intracranial mass lesion, case 2 is a 69-year-old man with diabetes, and case 3 is a 81-year-old man with cardiac arrest) at the department of human anatomy in Nanjing medical University in 2018. Fresh heart samples were collected and every specimen was analyzed by at least two experienced cardiac anatomists. After good exposure of the hearts, the coronary arteries were isolated and each coronary artery specimen was separated into 10 segments according to human anatomy: the left main trunk (LM), the proximal segment of the left anterior descending artery (LAD-P), the midsegment of the left anterior descending artery (LAD-M), the distal segment of the left anterior descending artery (LAD-D), the proximal segment of the left circumflex artery (LCX-P), the midsegment of the left circumflex artery (LCX-M), the distal segment of the left circumflex artery (LCX-D), the proximal segment of the right coronary artery (RCA-P), the midsegment of the right coronary artery (RCA-M), the distal segment of the right coronary artery (RCA-D). Every artery segments of case 1 and case 2 was divided into two groups in half: protein group and pathological group, and the artery segments of case 3 were divided into RNA group and pathological group likewise. Segments in the protein group and the RNA group were snapfrozen in liquid nitrogen and stored at $-80^{\circ} \mathrm{C}$ for backup purposes, and the segments in the pathological groups were fixed in $10 \%$ formalin overnight for further histological analysis.

\section{Clinical and pathologic analysis}

The segments in pathologic groups from 3 samples were fixed in 10\% formalin overnight. Decalcification using EDTA (ethylene diamine tetraacetic acid) decalcifying solution (Solarbio ${ }^{\circledR}$ LIFE SCIENCES, Beijing, China) was conducted for about half a months subsequently. H\&E (haematoxylin and eosin) staining was performed in the standardized laboratory in the pathology department of the First Affiliated Hospital of Nanjing Medical University. All of the pathological sections were observed under the optical microscope (Leica DM2500 Wien, Austria) and digitalized by the matched image analysis system (Leica LAS, Wetzlar, Germany). The rest of the samples were stored in wax blocks. The pathological staging of 
the slides was classified based on international standard. Lesions were scored as no lesion (NL) stage, lipid strain (LS) stage, fibrous plaque (FP) stage, atheromatous plaque (AP) stage and secondary lesion (SL) stage ${ }^{(8)}$.

\section{Protein exaction and quantification}

In this study, label-free quantification was conducted for proteomics analysis. All of the samples from case 1 and case 2 were ground in liquid nitrogen and transferred to $1.5 \mathrm{ml}$ centrifuge tubes perspectively. 400 ul of L3 lysis buffer, 1 mM PMSF, 2 mM EDTA, 10 mM DTT were added into the tube and were mixed with a pipette. Put the centrifuge tubes to ice bath to ultrasound for 15 minutes, followed by centrifugation for 20 minutes at $13000 \mathrm{~g}, 4^{\circ} \mathrm{C}$. The supernatant was precipitated with four-times the volume of cold acetone and $30 \mathrm{mM}$ DTT at $-20^{\circ} \mathrm{C}$ for 2 hours. Afterwards, $4^{\circ} \mathrm{C}, 13000 \mathrm{~g}$ centrifugation for 20 minutes was performed and the supernatant was discarded, and $1 \mathrm{ml}$ of cold acetone with $10 \mathrm{mM}$ DTT was added into the precipitate. After fully mashing and concussion, the precipitate was left at $-20^{\circ} \mathrm{C}$ for 30 minutes and centrifuged at $13000 \mathrm{~g}$ for $20 \mathrm{~min}$. The supernatant was discarded and the precipitate was added with $200 \mathrm{ul} \mathrm{M2}$ lysis buffer after air-drying. At last, put the centrifuge tubes to ice bath to ultrasound for 5 minutes and then centrifuge at $4^{\circ} \mathrm{C}, 13000 \mathrm{~g}$ for 20 minutes. Take the supernatant and determine the protein concentration using the Bradford method (Bradford 1976). The protein identification and quantification was conducted with ProteinPilot software (version 4.5, SCIEX, Redwood City, California, USA).

\section{RNA isolation and sequencing}

Extraction of total RNA in the prepared samples from case 3 was conducted with Trizol (15596018》 Invitrogen) according to the manufacturer's instructions. The quality control was performed and the RNA integrity was checked with Agilent Bioanalyzer 2100 (Agilent technologies, US). RNA purity and concentration were checked using the NanoPhotometer ${ }^{\circledR}$ spectrophotometer (IMPLEN, CA, USA). A total of 3 ug RNA was extracted from per sample for the further experiments. The ribosomal RNA was removed by Epicentre Ribo-zero ${ }^{\text {TM }}$ rRNA Removal Kit (Epicentre, USA), and rRNA free residue was cleaned up by ethanol precipitation. The sequencing libraries were generated using the rRNA-depleted RNA by NEBNext ${ }^{\circledR}$ Ultra ${ }^{\mathrm{TM}}$ Directional RNA Library Prep Kit for Illumina ${ }^{\circledR}$ (NEB, USA) following manufacturer's recommendations. The first strand CDNA was synthesized with random hexamer primer and M-MuLV Reverse Transcriptase, and the second strand CDNA was synthesized with DNA polymerase I and RNase $\mathrm{H}$. In the reaction buffer, dNTPs and dTTP were replaced by dUTP. The overhangs were converted into blunt ends via exonuclease/polymerase activities. After adenylation of 3 ' ends of DNA fragments, NEBNext Adaptor with hairpin loop structure were ligated to prepare for hybridization. The library fragments were purified and the cDNA fragments with 150 200 bp in length were selected with AMPure XP system (Beckman Coulter, Beverly, USA). 3 ul USER Enzyme (NEB, USA) was used to react with sizeselected, adaptor-ligated $\mathrm{CDNA}$ at $37^{\circ} \mathrm{C}$ for $15 \mathrm{~min}$, followed by $5 \mathrm{~min}$ at $95^{\circ} \mathrm{C}$. The PCR was performed subsequently and the products were purified on AMPure XP system. The library quality was assessed on the Agilent Bioanalyzer 2100 system $^{(8)}$. 


\section{Bioinformatics analysis}

All the identified proteins from case 1 and case 2 were divided into 5 groups according to the pathological grading. The relative quantification of the proteins in LS group, FP group, AP group and SL group was compared to that in NL group. The proteins with no expression were removed. Differencial expression was defined as fold change $>2$ or fold change $<0.5$ and the significantly differential expression proteins (SDEPs) were defined as proteins showed differencial expression in all of the 4 comparison groups. The identified RNAs in the RNA sequencing included mRNAs (messager RNAs), IncRNAs (long non-coding RNAs) and circRNAs (circular RNAs). The quantification of circRNAs was performed and TPM (Transcripts per million reads) normalization was conducted for further analysis. The normalized expression quantities of the circRNAs in LAD-P, LAD-M, LAD-D, LCX-P, LCX-M, LCX-D, RCA-P, RCA-M and RCA-D groups were compared to that in LM group respctively (the lesions were mainly located in the left main trunk in case 3). The significantly differential expression circRNAs (SDECs) were screened by the method above. The gene-protein interaction analysis was conducted using Targetscan Human 7.2 software. Gene ontology (GO) functional classification was performed by Blast2GO software (http://www.blast2go.de), and contigs were divided into biological processes, cellular components and molecular functions according to $\mathrm{GO}$ terms. The target binding sites prediction between mir-155 and circRNAs was performed using circinteractomedatabase (https://circinteractome.nia.nih.gov).

\section{Results}

\section{The pathologic analysis of the coronary samples}

The pathological stages of the samples were graded as NL stage, LS stage, FP stage, AP stage and SL stage according to the microscopic characteristics (Figure 1). In case 1, all the coronary branches were severely diseased. Lesions in LM, LCX-P, RCA-P were scored as SL stage and were severer than other branches. Lesions in case 2 were mostly presented in LM and LAD, while LCX and RCA were relatively slightly diseased. Case 3 was died of cardiac arrest resulted from LM obstruction. The slides under the microscope showed a deadly lesion in LM, while other branches seemed healthy (Table 1).

\section{Differential expression proteins and circRNAs by proteomics and transcriptomics}

A proteomics approach was used for protein analysis in our samples. 2135 protein groups were identified and 21703 unique peptides were included. Proteins identified from the peptides fragments of 20 coronary samples from case 1 and case 2 were divided into 5 groups according to the results of pathological analysis. 174 proteins were obtained as significant differential expression between diseased samples and controls. In a transcriptomics analysis, 1640 circRNAs were identified, including 66 significant differential expression circRNAs. In the light of the results of pathological analysis, 54 circRNAs showed upregulated in cases and 12 circRNAs showed downregulated. Part of the results have been published online. (https://www.ncbi.nlm.nih.gov/pmc/articles/PMC6895512/, https://www.spandidos-

publications.com/confirmCorrespondingEmail?

transactionld=3078312e37323038306533623463636331703430). 


\section{Bioinformatics analysis of the ceRNA network in coronary artery disease}

To clarify the molecular mechanism that mir-155 participate in the occurrence and develpoment of CAD, the bioinformatics analysis was conducted.

Functional annotation and enrichment analysis of 174 SDEPs were performed. GO (Gene Ontology) analysis showed that SDEPs mainly located in mitochondron (GO: 0005739), mitochondrial part (GO: 0044429), contractile fiber part (GO: 0044449), sarcomere (GO: 0030017), mitochondrial membrane part (GO: 0044455). Molecular Function mainly included "electron transfer activity (GO: 0009055)", "oxidoreductase activity (GO: 0016491)", "structural constituent of muscle (GO: 008307)", "actin binding (GO: 0003779)" and "actin filament binding (GO: 0051015)". Biological Process mainly included "small molecule metabolic process (G0:0044281)", "generation of precursor metabolites and energy (G0:0006091)", "drug metabolic process (GO:0017144)", "purine ribonucleoside triphosphate metabolic process (G0:0009205)" and "ATP metabolic process (G0:0046034)". The KEGG pathway analysis showed that the SDEPs mainly enriched in "cardiac muscle contraction (hsa04260)", "Parkinson's disease (hsa05012)", "Huntington's disease (05016)", "oxidative phosphorylation (hsa00190)" and "metabolic pathways (hsa01100)".

A protein-protein interaction (PPI) analysis was conducted using the String Database (https://string$\mathrm{db}$. org/cgi/input.pl) and results were showed in Figure 2. Targetscan Human 7.2 software was used to screen the downstream target genes of mir-155 and 556 protein coding mRNAs with potential binding sites of mir-155 were obtained. After taking the intersection with SDEPs, 2 mRNAs and their coding proteins were identified, including GPD1L (glycerol-3-phosphate dehydrogenase 1 like) (Table 2) and BCAT1 (branched-chain aminotransferase 1) (Table 3). Among the PPI analysis, GPD1L and BCAT1 interacted with plenty of the identified SDEPs and may play an important role in CAD (Figure 2). As was shown in Figure 3, the quantification of GPD1L showed a continuous decrease as the lesion getting severer. Figure 4 showed the quantification of BCAT1 in samples of different pathological stages.

To find the putative regulatory circRNA located upstream of mir-155, circinteractome database (https://circinteractome.nia.nih.gov) was used to screen the miRNA binding sites from the 66 candidate circRNAs. 5 circRNAs including hsa_circ_0004182, hsa_circ_0004617, hsa_circ_0005030, hsa_circ_0013167 and hsa_circ_002595, were predicted to be target combined with mir-155. Among the 5 circRNAs, 6 binding sites were found between mir-155 and hsa_circ_0004182, which was downregulated in grossly diseased samples (Table 4).

Based on the above researches, a ceRNA network was predicted to play a regulatory role in CAD (Figure $5)$.

\section{Conclusion}

A ceRNA network may play an important role in the occurence and development of CAD. In these genes, hsa_circ_0004182 may inhibit the expression of mir-155 by acting as miRNA sponges, and repress the 
post-transcriptional repression of GPD1L mediated by mir-155, and promote GPD1L expression in coronary artery disease.

\section{Discussion}

In our previous study, circulating mir-155 in plasma showed a significant differential expression in CAD populations and controls ${ }^{(6)}$. For further study, we detected the expression of circRNAs and proteins in human coronary artery samples with genome sequencing. 2135 protein groups, 21703 unique peptides and 1640 circRNAs were identified in the samples. 174 differential expressed proteins and 66 differential expressed circRNAs were screened according to the pathological grading and staging of the samples ${ }^{(8)}$.

The gene-gene interaction and gene-protein interaction analysis showed that a novel ceRNA network may play a regulatory role in CAD. And the miRNA target prediction suggested that mir- 155 , which is targeted by hsa_circ_0004182, may play a role in CAD by repressing the expression of GPD1L.

GPD1L is aslo known as glycerol-3-phosphate dehydrogenase 1 like. The protein encoded by the GPD1L gene catalyzes the synthesis of glycerone phosphate and is related to. Defects in this gene has been recognized as the cause of Brugada syndrome type 2 (BRS2) as well as sudden infant death syndrome $(\operatorname{SIDS})^{(9,10)}$.

GO analysis showed that the SDEPs were significantly associated with metabolism, oxidative respiration, and mitochondrial function. KEGG pathway enrichment analysis showed that the SDEPs were mostly enriched in metabolic pathways and neuropathic pathways including Huntington's disease and Parkinson's disease.

Studies showed that the abnormal protein accumulating and protein cytotoxicity acted as important initial factors resulting in Altheimer's disease, Huntington's disease, and Parkinson's disease. Besides, it was also reported that protein degradation relied on proteasome and autophagolysosome via ubiquitination pathway, and aotuphagy played an important role in this biological process ${ }^{(11)}$. Based on the results of experiments and bioinformatics analysis, we speculated that hsa_circ_0004182 targeting mir-155 regulates mitophagy via promoting the expression of GPD1L in coronary artery disease.

Mitochondria are important organelles involved in energy metabolism in the cell. In addition to providing ATP that maintains cell activity and function through the oxidation respiratory chain, they also participate in various physiological processes such as the generation of reactive oxygen species and the regulation of calcium homeostasis. Mitophagy eliminates damaged mitochondria and it is of great significance for maintaining intracellular homeostasis in cardiomyocytes, endothelial cells, and smooth muscle cells ${ }^{(12)}$.

Studies showed that the inhibition of mitophagy will aggravate myocardial ischemia-reperfusion injury. For example, Lu W knocked out the Pgam5 gene in myocardial ischemia mice model and found that the myocardial infarction area turned larger than that in the controls ${ }^{(13)}$. Kubli DA revealed that the 
myocardial damage was much severer in mitophagy-deficient mice than that in controls after permanent ligation of $L A D^{(14)}$.

In this study, we found a potential ceRNA network in the regulation of the occurrence and development of CAD. Besides, we put forward the hypothesis that hsa_circ_0004182 targeting mir-155 regulates mitophagy via promoting the expression of GPD1L in coronary artery disease. Our study provided useful ideas for searching new autophagy-related proteins and for exploring the molecular mechanism of mitophagy. However, the experimental veritification of the interactions among hsa_circ_0004182, mir-155 and GPD1L is lacking, and further study is required.

\section{Declarations}

\section{Ethical approval and consent to participate}

The next of kin of the patients from which the samples were taken from gave explicit consent for the samples to be used in this study. Written informed consent was obtained from the bereaved families for the publication of any potentially identifiable images or data included in this article.All the experimental protocols were approved by the ethics committee of the Nanjing Medical University.

\section{Consent to publish}

All authors have read and approved the manuscript. We certify that we have each made a substantial contribution so as to qualify for authorship and that we have approved the contents.

\section{Availability of data and materials}

All the supporting data are available.

\section{Competing interests}

There are no potential conflicts of interests in this work.

\section{Funding}

This study received support from the National Natural Science Foundations of China (NO:81970302).

\section{Authors' contributions}

As the guarantor, Enzhi Jia conceived the study. Qiaowei Jia and Wenfeng Ji initially drafted the paper. Chenhui Zhao enrolled participants and collected the data under the supervision of Enzhi Jia, Yaqing Zhou cleaned and analysed the data. Can Hou monitored the conduct of the study and reviewed the safety and effectiveness data. All authors reviewed the manuscript.

\section{Acknowledgements}


Not applicable

\section{Authors' Information}

Authors:

Qiaowei Jia ${ }^{2}$, Wenfeng $\mathrm{Ji}^{1}$, Chenhui Zhao ${ }^{1}$, Yaqing Zhou ${ }^{1}$, Can Hou ${ }^{1}$, Enzhi Jia ${ }^{1,}$

Affiliations:

${ }^{1}$ Department of Cardiovascular Medicine, The First Affiliated Hospital of Nanjing Medical University, Nanjing210029, Jiangsu Province, China.

${ }^{2}$ Department of Cardiovascular Medicine, Liyang People's Hospital, Liyang213300, Jiangsu Province, China.

*Jia QW and Ji WF contributed to the work equally.

*Corresponding Author: Enzhi Jia, MD.

\section{References}

1. World Health Statistics 2017: Monitoring health for the SDGs. WHO. 17 MAY 2017.

2. Khera AV, Kathiresan S. Genetics of coronary artery disease: discovery, biology and clinical translation. Nat Rev Genet. 2017 Jun;18(6):331-344.

3. Gertler MM, Garn SM, White PD. Young candidates for coronary heart disease. J Am Med Assoc. 1951;147:621-625.

4. Musunuru K, Kathiresan S. Genetics of Common, Complex Coronary Artery Disease. Cell. 2019 Mar 21;177(1):132-145.

5. Lu TX, Rothenberg ME. MicroRNA. J Allergy Clin Immunol. 2018 Apr;141(4):1202-1207.

6. Jia QW, Chen ZH, Ding XQ, Liu JY, Ge PC, An FH, Li LH, Wang LS, Ma WZ, Yang ZJ, Jia EZ. Predictive Effects of Circulating miR-221, miR-155 and miR-130a for Coronary Heart Disease: A Multi-Ethnic Study in China. Cell Physiol Biochem. 2017;42(2):808-823.

7. Zhang F, Zhao J, Sun D, Wei N. MiR-155 inhibits transformation of macrophages into foam cells via regulating CEH expression. Biomed Pharmacother. 2018;104:645-651.

8. Pan RY, Zhao CH, Yuan JX, Zhang YJ, Jin JL, Gu MF, Mao ZY, Sun HJ, Jia QW, Ji MY, Zhang J, Wang LS, Ma WZ, Ma WQ, Ding JD, Jia EZ. Circular RNA profile in coronary artery disease. Am J Transl Res. 2019 Nov 15;11(11):7115-7125. (https://pubmed.ncbi.nlm.nih.gov/31814914/)

9. Hedley PL, Jørgensen P, Schlamowitz S, Moolman-Smook J, Kanters JK, Corfield VA, Christiansen M. The genetic basis of Brugada syndrome: a mutation update. Hum Mutat. 2009 Sep;30(9):1256-66. 
10. Valdivia CR, Ueda K, Ackerman MJ, Makielski JC. GPD1L links redox state to cardiac excitability by PKC-dependent phosphorylation of the sodium channel SCN5A. Am J Physiol Heart Circ Physiol. 2009 Oct;297(4):H1446-52.

11. Doyle $S M \otimes G e n e s t ~ O \otimes W i c k n e r ~ S$. Protein rescue from aggregates by powerful molecular chaperone machines. Nat Rev Mol Cell Biol. 2013 Oct;14(10):617-29.

12. Bravo-San Pedro JM, Kroemer G, Galluzzi L. Autophagy and Mitophagy in Cardiovascular Disease. Circ Res. 2017;120(11):1812-1824.

13. Lu W, Sun J, Yoon JS, Zhang Y, Zheng L, Murphy E, Mattson MP, Lenardo MJ. Mitochondrial protein PGAM5 regulates mitophagic protection against cell necroptosis.PLoS One. 2016;11:e0147792.

14. Kubli DA, Zhang X, Lee Y, Hanna RA, Quinsay MN, Nguyen CK, Jimenez R, Petrosyan S, Murphy AN, Gustafsson AB. Parkin protein deficiency exacerbates cardiac injury and reduces survival following myocardial infarction.J Biol Chem. 2013;288:915-926.

\section{Tables}

Table 1. The results of pathological analysis in 3 cases

\begin{tabular}{|llll|}
\hline & Case 1 & Case 2 & Case 3 \\
\hline LM & SL & AP & SL \\
LAD-P & AP & AP & LS \\
LAD-M & AP & AP & LS \\
LAD-D & AP & NL & FP \\
\hline LCX-P & SL & NL & FP \\
\hline LCX-M & AP & LS & FP \\
\hline LCX-D & AP & LS & LS \\
RCA-P & SL & FP & LS \\
RCA-M & AP & AP & LS \\
\hline RCA-D & AP & LS & FP \\
\hline
\end{tabular}

Table 2. The binding site prediction between mir-155 and GPD1L 
Position 407-414 of GPD1L $\quad 5^{\prime} \ldots .$. UGUUAAAUUUUAACCAGCAUUAA....

3'UTR

hsa-miR-155-5p

3' UGgGGAUAGUGCUAAUCGUAAUU

Table 3. The binding site prediction between mir-155 and BCAT1.

\begin{tabular}{|llc|}
\hline & $\begin{array}{l}\text { Predicted consequential pairing of target region and } \\
\text { miRNA }\end{array}$ & Site type \\
\hline $\begin{array}{l}\text { Position 2573-2579 of BCAT1 } \\
\text { 3'UTR }\end{array}$ & 5'....GGCAUAAGAUGGUCUAGCAUUAG.... & \\
\hline hsa-miR-155-5p & 3' UGGGGAUAGUGCUAAUCGUAAUU & m8 - \\
\hline
\end{tabular}

Table 4. The binding sites prediction between mir-155 and hsa_circ_0004182

\begin{tabular}{|c|c|c|c|}
\hline CircRNA MirBase ID & CircRNA-miRNA pairing & Sites Start & Sites End \\
\hline hsa_circ_0004182 (5'...3') & CACAUUCUGGCAAGAAGCAUUAA & 17110 & 17117 \\
\hline hsa-miR-155-5p (3'...5') & UGGGGAUAGUGCUAAUCGUAAUU & & \\
\hline hsa_circ_0004182 (5'...3') & AAAACAUGUAAAUGGGCAUUAAA & 18518 & 18524 \\
\hline hsa-miR-155-5p (3'...5') & UGGGGAUAGUGCUAAUCGUAAUU & & \\
\hline hsa_circ_0004182 (5'...3') & CUCAAGUGUGCCUAGAGCAUUAG & 24716 & 24722 \\
\hline hsa-miR-155-5p (3'...5') & UGGGGAUAGUGCUAAUCGUAAUU & & \\
\hline hsa_circ_0004182 (5'...3') & CCAAUAACAGUGUCUGCAUUAAC & 415 & 421 \\
\hline hsa-miR-155-5p (3'...5') & UGGGGAUAGUGCUAAUCGUAAUU & & \\
\hline hsa_circ_0004182 (5'...3') & GAUUUGUUUUCAAUA-AGCAUUAU & 8915 & 8921 \\
\hline hsa-miR-155-5p (3'...5') & UGGGGAUAGUGCUAAUCGUAAUU & & \\
\hline hsa_circ_0004182 (5'...3') & UUUGAGGCUGCUCUCAGCAUUAC & 9280 & 9286 \\
\hline hsa-miR-155-5p (3'...5') & UGGGGAUAGUGCUAAUCGUAAUU & & \\
\hline
\end{tabular}




\section{Figures}

\section{LAD-D in case 2: NL stage LAD-M in case 3: LS stage}

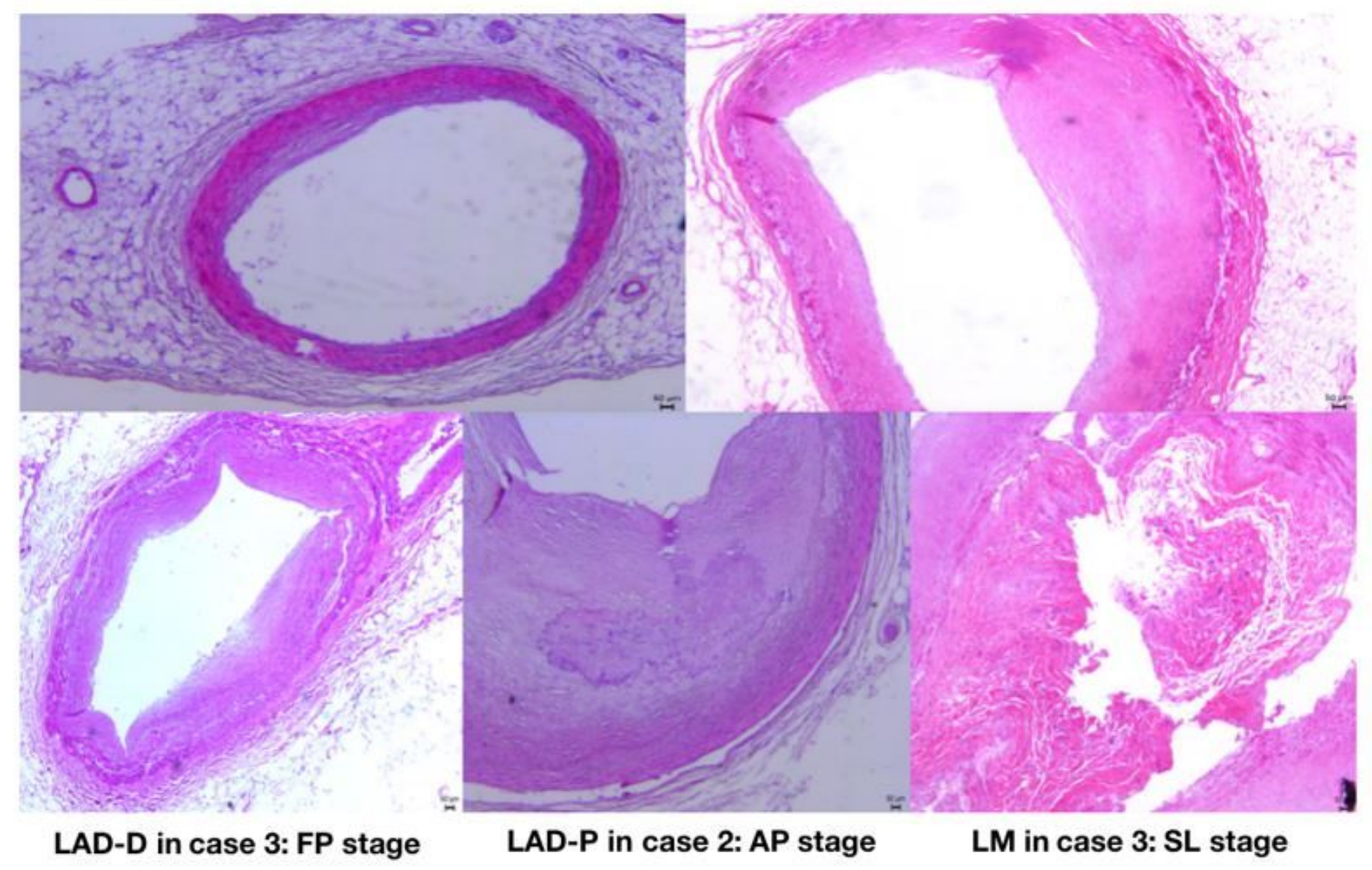

Figure 1

The microscopic characteristics of the different pathological stages 


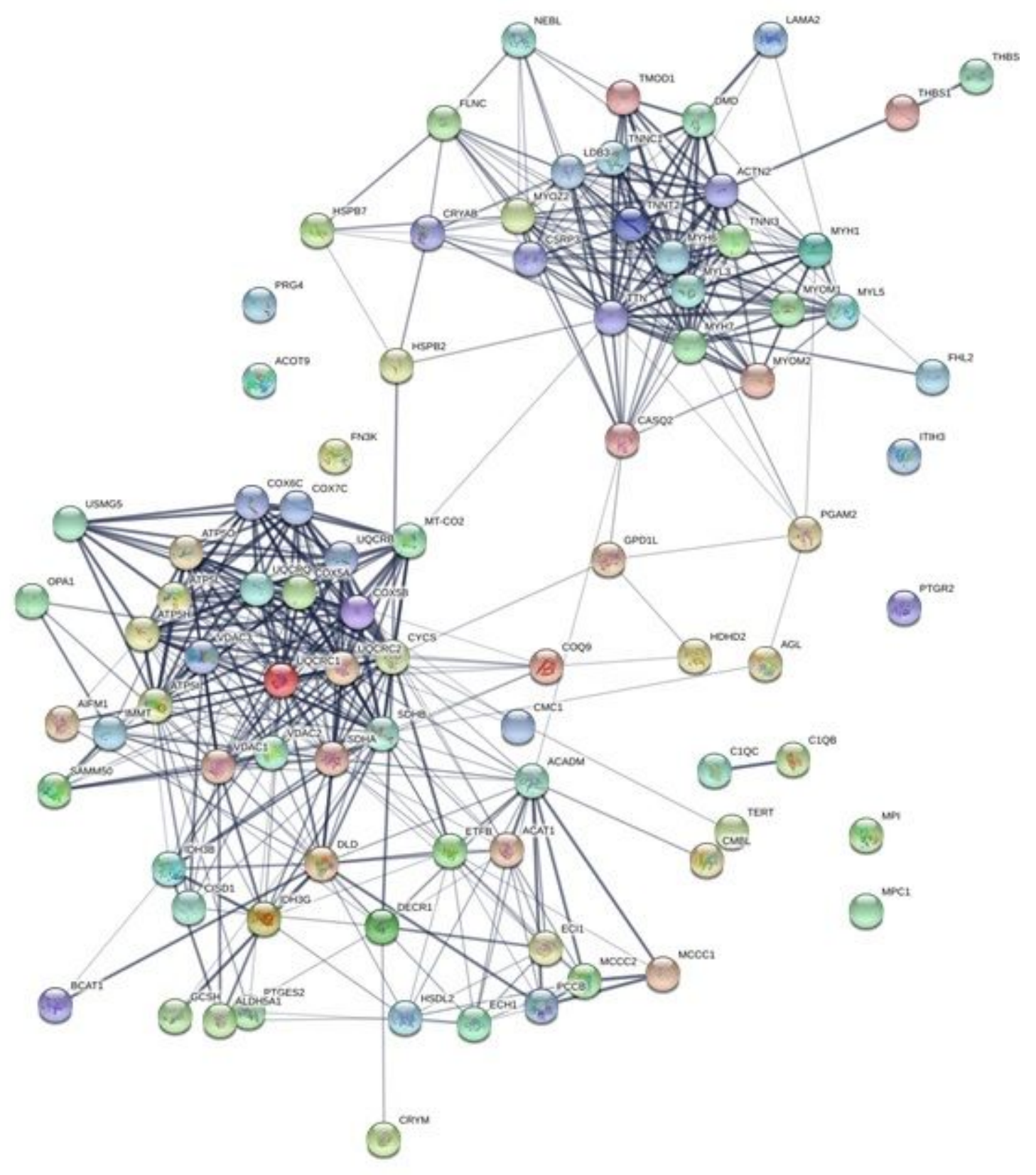

Figure 2

The protein-protein interaction network by the type of interaction evidence Line thickness indicates the strength of data support. 


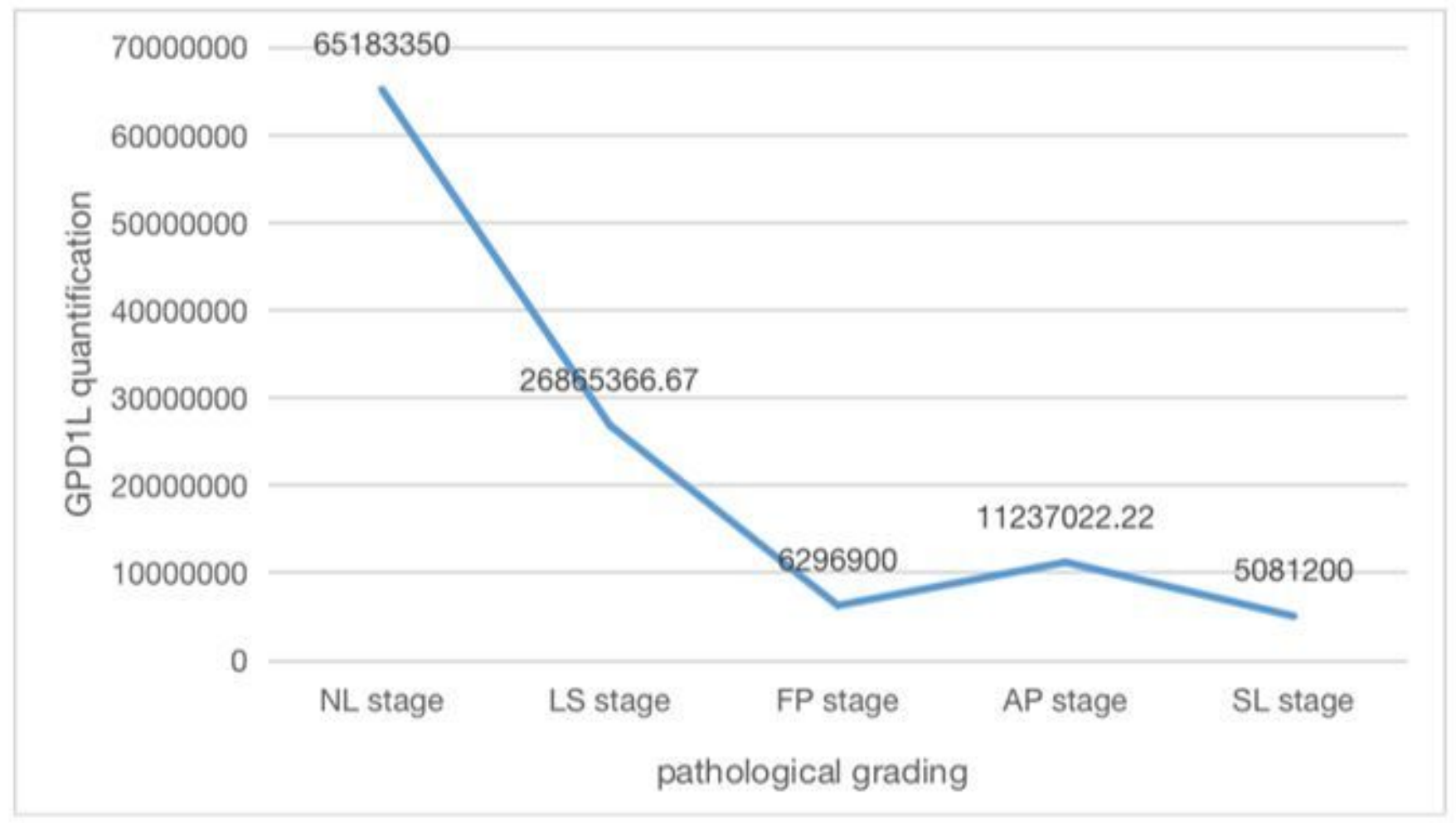

\section{Figure 3}

GPD1L quantification in samples of different pathological stages

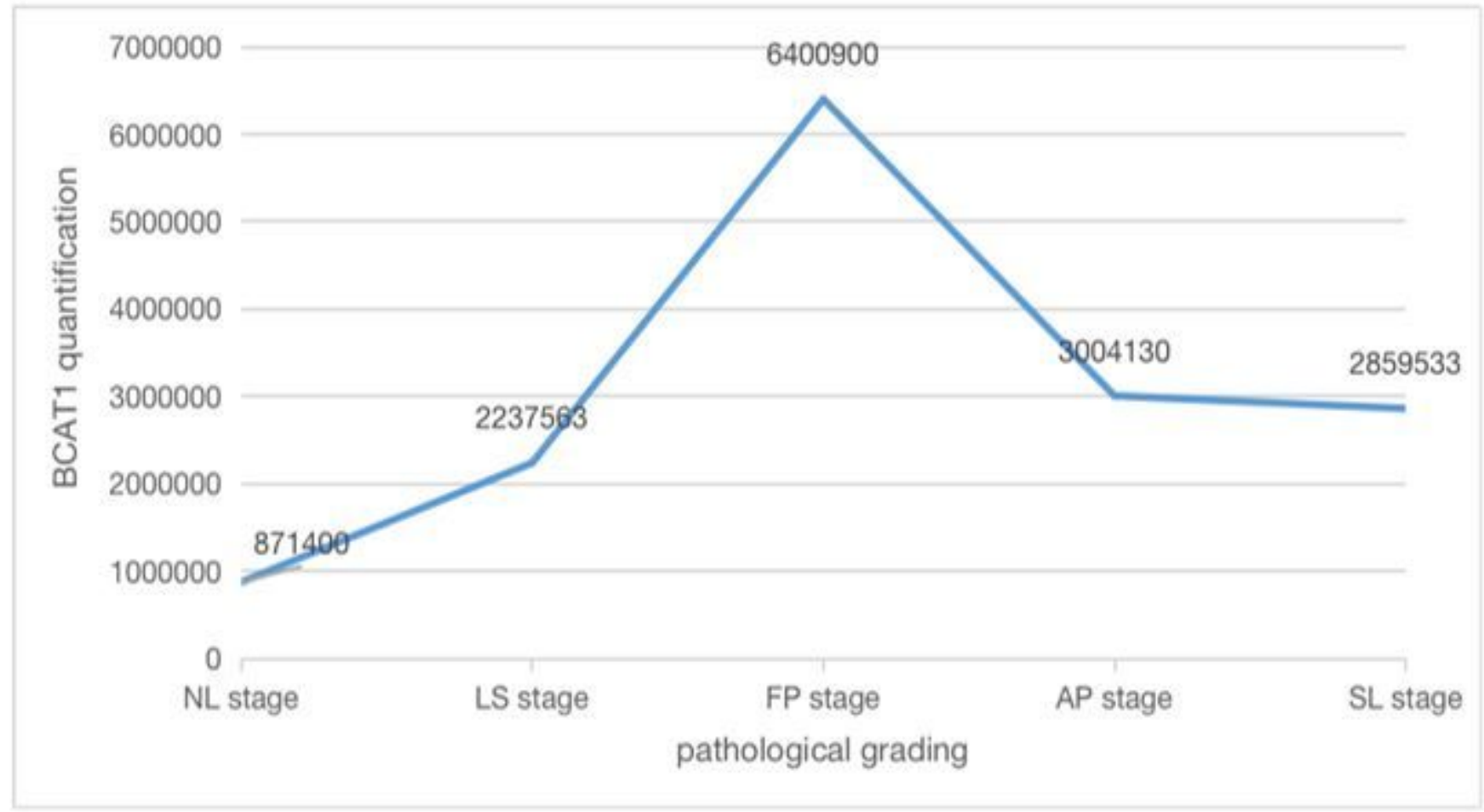

Figure 4

BCAT1 quantification in samples of different pathological stages 


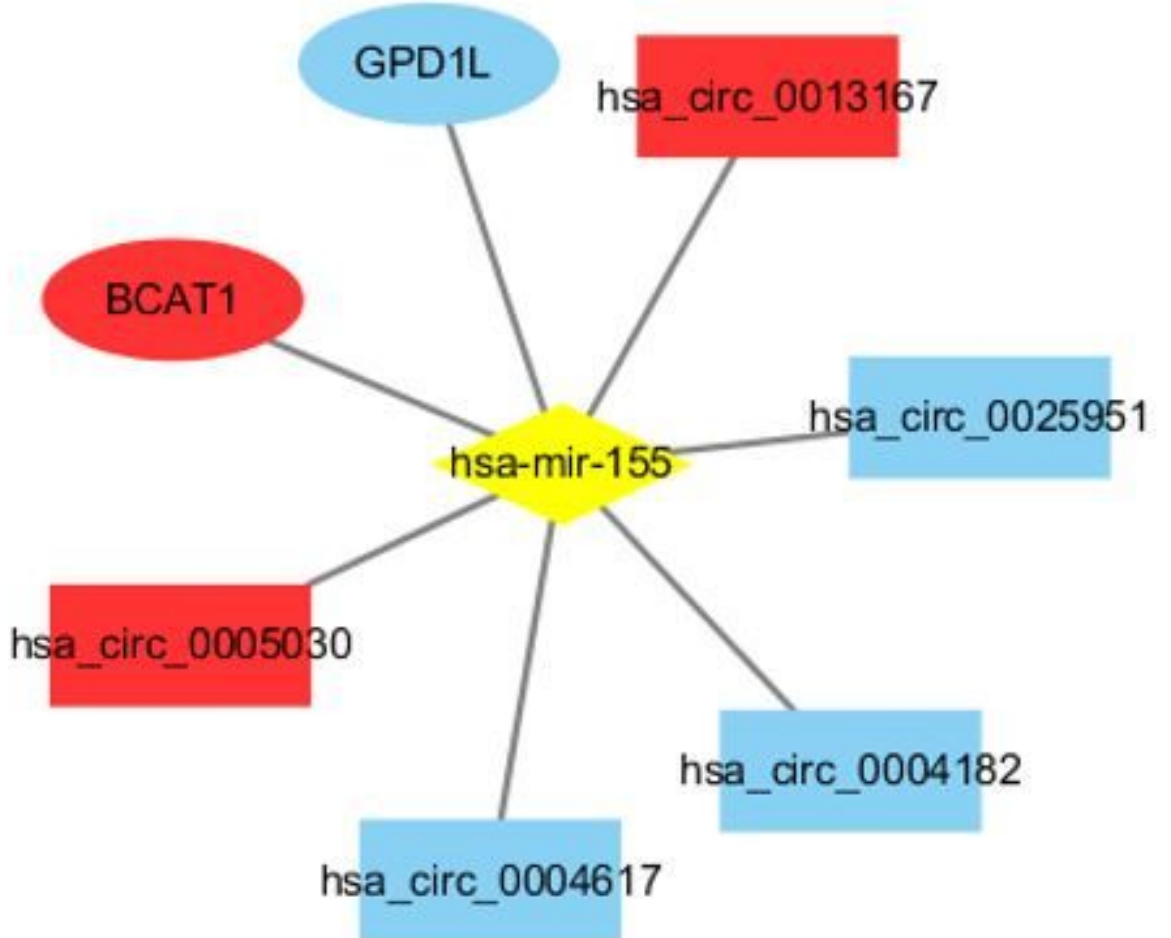

Figure 5

The predicted ceRNA network in CAD The different shapes represent circRNA, miRNA and mRNA respectively. The genes in red blankets are up-regulated in CAD group, and these in blue blankets are down-regulated.

\section{Supplementary Files}

This is a list of supplementary files associated with this preprint. Click to download.

- supplementarydata1.xlsx

- supplementarydata2.xls 